\title{
Five-year clinical results of cervical total disc replacement compared with anterior discectomy and fusion for treatment of 2-level symptomatic degenerative disc disease: a prospective, randomized, controlled, multicenter investigational device exemption clinical trial
}

\author{
Kris Radcliff, MD, ${ }^{1}$ Domagoj Coric, $M D,{ }^{2}$ and Todd Albert, MD $^{3}$ \\ ${ }^{1}$ Department of Orthopedic Surgery, Thomas Jefferson University, Rothman Institute, Egg Harbor, New Jersey; ${ }^{2}$ Carolinas \\ Medical Center, Carolina Neurosurgery \& Spine Associates, Charlotte, North Carolina; and ${ }^{3}$ Hospital for Special Surgery, Weill \\ Cornell Medical College, New York, New York
}

OBJECTIVE The purpose of this study was to report the outcome of a study of 2-level cervical total disc replacement (Mobi-C) versus anterior cervical discectomy and fusion (ACDF). Although the long-term outcome of single-level disc replacement has been extensively described, there have not been previous reports of the 5-year outcome of 2-level cervical disc replacement.

METHODS This study reports the 5-year results of a prospective, randomized US FDA investigational device exemption (IDE) study conducted at 24 centers in patients with 2-level, contiguous, cervical spondylosis. Clinical outcomes at up to 60 months were evaluated, including validated outcome measures, incidence of reoperation, and adverse events. The complete study data and methodology were critically reviewed by 3 independent surgeon authors without affiliation with the IDE study or financial or institutional bias toward the study sponsor.

RESULTS A total of 225 patients received the Mobi-C cervical total disc replacement device and 105 patients received ACDF. The Mobi-C and ACDF follow-up rates were $90.7 \%$ and $86.7 \%$, respectively ( $p=0.39$ ), at 60 months. There was significant improvement in all outcome scores relative to baseline at all time points. The Mobi-C patients had significantly more improvement than ACDF patients in terms of Neck Disability Index score, SF-12 Physical Component Summary, and overall satisfaction with treatment at 60 months. The reoperation rate was significantly lower with Mobi-C (4\%) versus ACDF (16\%). There were no significant differences in the adverse event rate between groups.

CONCLUSIONS Both cervical total disc replacement and ACDF significantly improved general and disease-specific measures compared with baseline. However, there was significantly greater improvement in general and disease-specific outcome measures and a lower rate of reoperation in the 2-level disc replacement patients versus ACDF control patients. Clinical trial registration no. NCT00389597 (clinicaltrials.gov) http://thejns.org/doi/abs/10.3171/2015.12.SPINE15824

KEY WORDS cervical disc herniation; cervical radiculopathy; cervical disc replacement; cervical arthroplasty

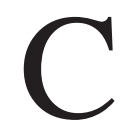
ERVICAL total disc replacement (cTDR) in properly indicated patients with single-level cervical disc pathology has been demonstrated to achieve satisfactory neural decompression and clinical outcomes equivalent to or better $4,6,7,14-28,38$ than anterior cervical discectomy and fusion (ACDF) in 7 different prospective, randomized, controlled US FDA trials. Single-level long- term data have been published on the Bryan disc, ${ }^{34}$ ProDisc-C, ${ }^{41}$ and Prestige ST disc, ${ }^{8}$ but this is the first report on the 5-year investigational device exemption (IDE) data for 2-level cervical arthroplasty.

Although both ACDF and cTDR satisfactorily treat clinically symptomatic cervical pathology, arthrodesis alters cervical mechanics ${ }^{1,2,11}$ by placing increased stresses on ad-

ABBREVIATIONS ACDF = anterior cervical discectomy and fusion; cTDR = cervical total disc replacement; IDE = investigational device exemption; MCS = Mental Component Summary; NDI = Neck Disability Index; PCS = Physical Component Summary; ROM = range of motion; SF-12 = 12-Item Short Form Health Survey; VAS = visual analog scale.

SUBMITTED July 10, 2015. ACCEPTED December 14, 2015.

INCLUDE WHEN CITING Published online March 25, 2016; DOI: 10.3171/2015.12.SPINE15824. 
jacent segments, which may contribute to the development of symptomatic degeneration at those adjacent levels. . $^{6,14,15,18}$ By preserving the motion of the operated segment, cTDR places comparatively less stresses on adjacent levels, which may serve to protect those levels. ${ }^{13,32,41}$ Multilevel ACDF is biomechanically more demanding than single-level ACDF with concomitant greater stress distribution on adjacent levels. ${ }^{3,11,26}$ Additionally, reoperation rates are generally higher in multilevel versus single-level ACDF. ${ }^{17}$ Currently there is a paucity of Level I evidence evaluating the outcome of multilevel cTDR procedures. . $17,27^{27}$

Mobi-C (LDR Medical) is a low-profile, mobile-bearing cTDR device composed of 2 cobalt-chromium alloy endplates and an ultra-high-molecular-weight polyethylene mobile core, resulting in a metal-on-polyethylene bearing surface. In April 2006, a large-scale, prospective, randomized, multicenter study was initiated at 24 sites comparing cTDR to ACDF in patients who had symptomatic cervical spondylosis at 2 contiguous levels. Davis et al. reported that at 2 years, the cTDR was associated with improved clinical success, greater reductions in Neck Disability Index (NDI) score, successful preservation of motion, and lower reoperation rate. ${ }^{12}$

The purpose of this study was to report the 5-year results of an IDE study of cTDR versus ACDF for the treatment of symptomatic 2-level contiguous cervical degenerative disc disease.

\section{Methods \\ Independent Review}

We received the complete 5-year data set via electronic file transfer to analyze and draft this paper. This data set included all relevant baseline parameters as well as outcomes at each follow-up visit through 5 years. The worksheets were presented as .txt files generated via source code and output from the SAS database (SAS Institute) in accordance with the statistical analysis plan. No data were omitted or redacted by the sponsor. All study methodology was reviewed in conjunction with the study results. As the author team, we retained final authority for the content of the paper, including the discussion and conclusions. We were provided with the study protocol, data analysis plan, and all corresponding study data. We were given the opportunity to review the entire scope of data analysis conducted and were provided complete information and data relevant to the scope of this paper.

\section{Study Design}

The study design has been described previously in detail. ${ }^{12}$ In brief, this was a prospective, randomized multicenter study at 24 clinical sites between April 2006 and March 2008 comparing the outcome of cTDR (Mobi-C) versus $\mathrm{ACDF}$. The control group underwent $\mathrm{ACDF}$ with a corticocancellous allograft and an anterior cervical plate. Investigators were blinded up to time of the procedure because the investigator needed to be prepared to perform either the treatment or control surgery. Patients were unblinded after surgery because the postoperative protocol varied between the 2 treatments, and it was not possible to prevent patients from viewing their own radiographs. This study was registered with the clinicaltrials.gov database (registration no. NCT00389597).

\section{Study Population}

Patients had a diagnosis of radiculopathy or myeloradiculopathy at 2 contiguous levels from C-3 to C-7 that was unresponsive to nonoperative treatment for at least 6 weeks or demonstrated progressive symptoms necessitating immediate surgery (Table 1). Exclusion criteria included any prior cervical spine surgery (Table 1). The protocol specified a sample size of 196 cTDR subjects and 98 control subjects based on a projected $60 \%$ control success rate and $65 \%$ cTDR success rate, and $80 \%$ power for a 1 -sided 0.05 significance level. Anticipated $10 \%$ loss-to-follow-up brought the total planned randomized sample size to 218 in the cTDR group and 109 in the ACDF control group. The study allowed for 1 nonrandomized training case per site. The nonrandomized patients were not included in the results of this paper.

Assuming that $2 \%$ of additional cases are lost per year, the power would be $99 \%$ for the overall success analysis.

\section{Treatment Assignment}

Three hundred thirty patients were randomized into the investigational device or the control group in a 2:1 ratio, resulting in 225 patients in the cTDR group and 105 in the ACDF group (Fig. 1). ${ }^{12}$ A 2:1 randomization design has been suggested as a way to improve the cost-effectiveness of clinical trials when there is a notable difference in cost between treatments. An unbalanced randomization is also appropriate as a way to increase patient access to a new therapy, especially when the outcomes of the control therapy are well understood. The sample size required to test the $10 \%$ noninferiority hypothesis of the study treatment to the control was powered assuming 2:1 randomization. This randomization method met the Oxford Centre for Evidence-Based Medicine's criteria and the North American Spine Society's criteria for Level 1 evidence. Thus, the 2:1 randomization method improved the cost-effectiveness of the study and allowed a greater amount of data to be gathered on the investigational device, without sacrificing statistical power. Please see CONSORT Criteria for Methods Section (Appendix 1).

\section{Follow-Up Routine}

Patients were evaluated preoperatively and at 1.5, 3, 6, $12,18,24,36,48$, and 60 months postoperatively. A total of 347 patients were randomized in the study, including 232 receiving cTDR and 115 receiving ACDF. Of these 347 patients, 225 in the cTDR group and 105 in the ACDF group received study surgery, with 17 patients withdrawing prior to surgery. ${ }^{12}$ There were no patients who crossed over from one group to another during the study surgery. Therefore, the primary randomized analysis population included 225 cTDR patients and 105 ACDF patients. There were no statistically significant differences between treatment groups (Table 2) in terms of demographic factors or baseline outcome measures (Table 3). The cTDR and ACDF follow-up rates were $90.7 \%$ and $86.7 \%$, respectively $(p=0.39)$, at 5 years. These follow-up rates include 
TABLE 1. Inclusion and exclusion criteria

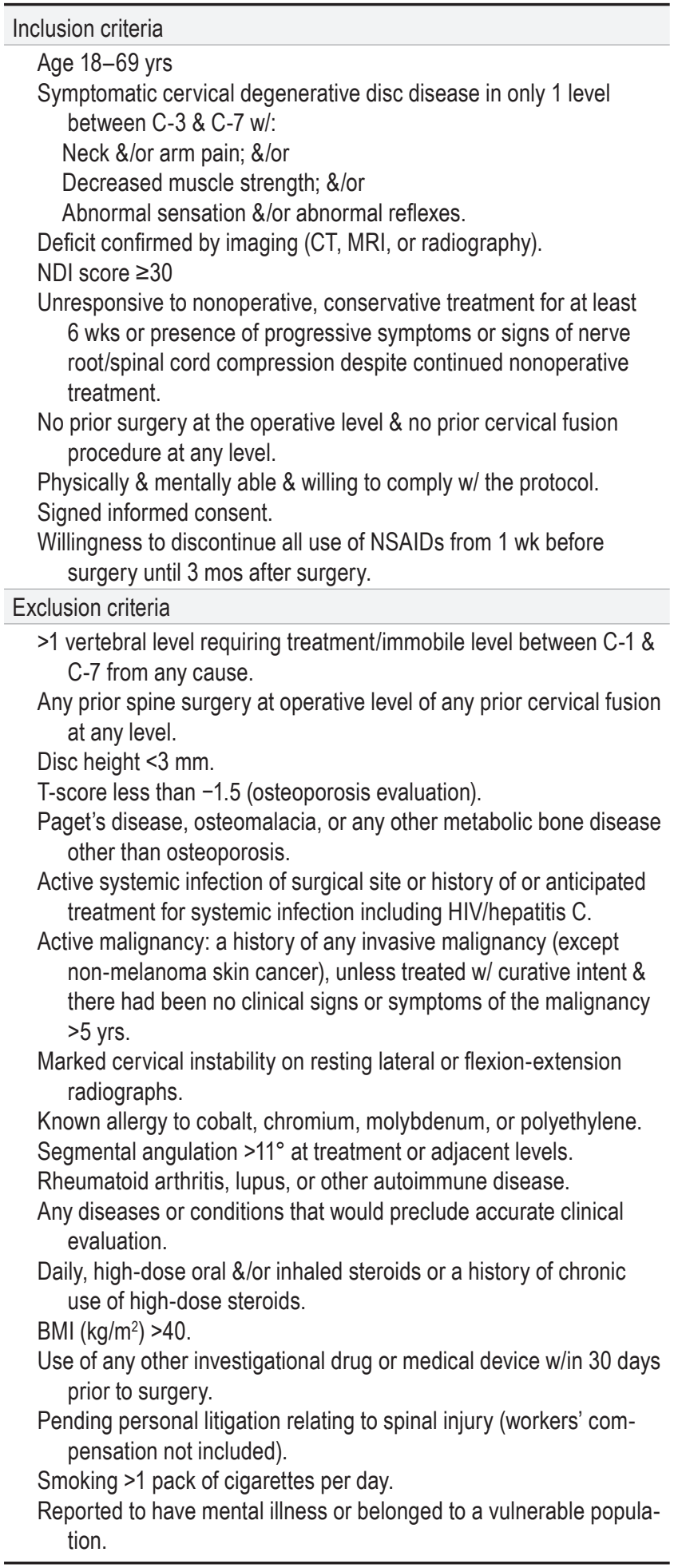

NSAID = nonsteroidal antiinflammatory drug.

patients with any data available at the 5-year visit. The follow-up rates reported in the paper are based on the number of patients eligible for follow-up at 5 years and do not include patients who dropped out earlier in the study (e.g., due to death, device failures). Those patients who did not contribute to the 5-year follow-up are still considered active in the study and will continue to be followed going forward.

\section{Outcome Measures}

Outcome scores included NDI, visual analog scale (VAS) scores for neck pain and arm pain, patient satisfaction with treatment, patient recommendation for same treatment, quality of life (12-Item Short Form Health Survey [SF-12]), and dysphagia. Additional clinical parameters were also analyzed as outcome measures, including the neurological assessments of strength, reflex, and motor testing. Fusion status was assessed radiographically. Lack of fusion was defined as $\geq 2^{\circ}$ of segmental movement on lateral flexion-extension radiographs, radiolucent lines at greater than $50 \%$ of the graft-vertebral interfaces, or lack of evidence of bridging trabecular bone. The study protocol did not specify the indications for reoperation in either treatment group. The decision to reoperate was determined solely by the treating surgeon and the patient's personal decision to proceed. A subsequent surgical intervention in either treatment group was strictly defined as a revision, removal, reoperation, or supplemental fixation at the index level.

Additionally, there was a composite definition for success similar to that used in other cervical arthroplasty device trials. ${ }^{12}$ In this analysis the end point was defined with the approval of the FDA within the Mobi-C Post-Approval Studies) protocol. The previously published definition of overall success by Davis et al. has been altered within the Post-Approval Studies protocol to include only clinical end points.

Overall study success was defined as requiring all 5 of the following metrics: 1) NDI improvement of at least 15 points (out of 50) from baseline; 2) No subsequent surgical intervention at the index level or levels; 3 ) No potentially (possibly or probably) device-related adverse event; 4) Maintenance or improvement in all components of neurological status; and 5) No Mobi-C intraoperative changes in treatment.

As previously reported, ${ }^{12}$ adverse events were defined and evaluated for relatedness in a blinded fashion to the treatment device when possible. In the tables, means are presented \pm SD.

\section{Results Neck Disability Index}

Patients in both groups showed significant improvement in NDI scores from baseline at all time points (Fig. 2 and Table 4). The mean improvement in NDI ( $p=0.0003)$ at 5 years was greater in the cTDR group (-37, SD 20) versus the ACDF group (-28, SD 18).

\section{Neck and Arm Pain}

Patients in both groups showed marked improvement from baseline at all time points. There was more improvement in VAS neck pain and VAS arm pain in the cTDR group than the ACDF group, although the difference was not statistically significant (Tables 5 and 6). 


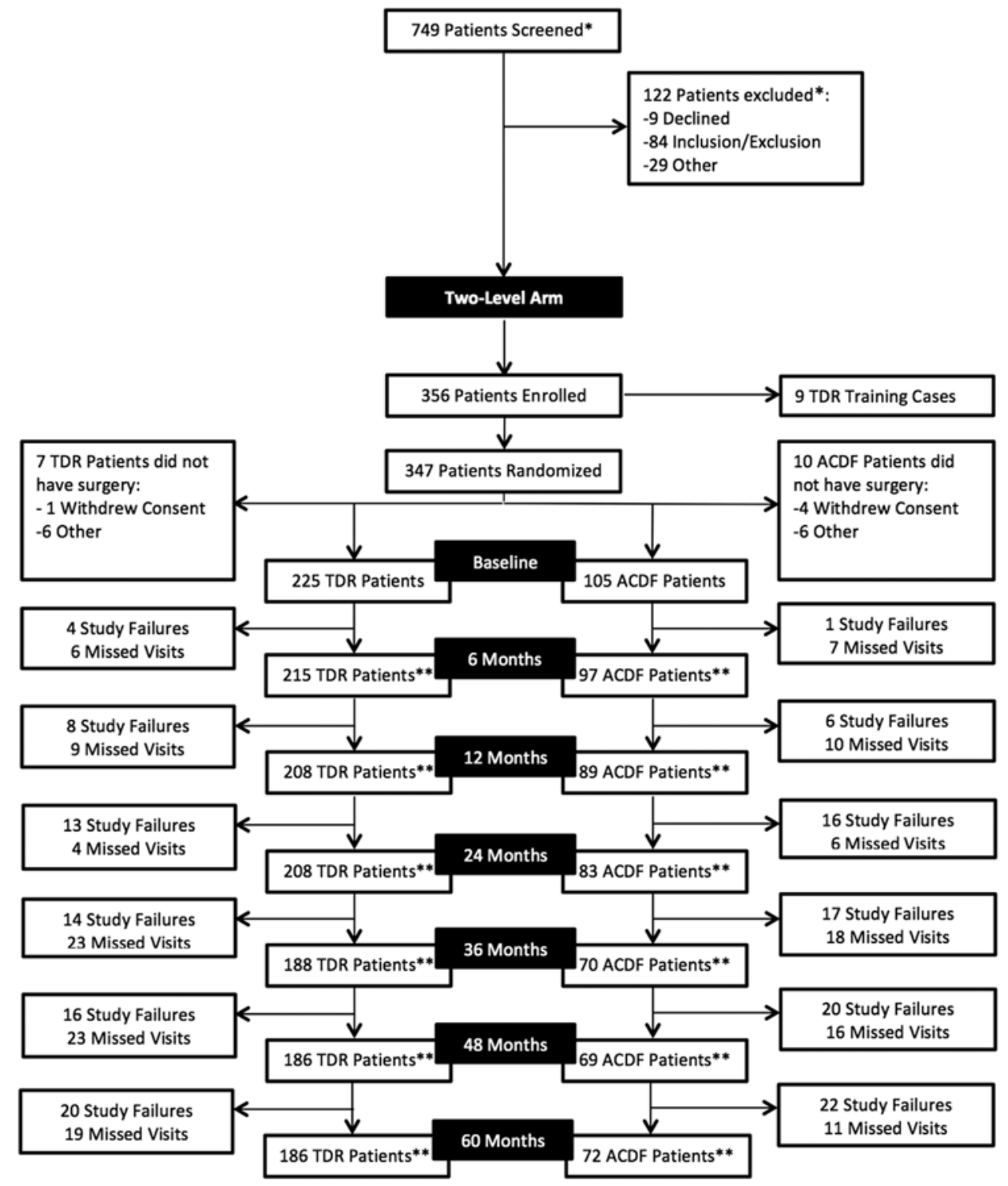

FIG. 1. CONSORT flow chart. *Patients were screened as part of the 1- or 2-level arm. ** Patients with any data available at the time point.

\section{Patient Satisfaction}

In both groups, the overall rates of patient satisfaction were high. However, there was significantly higher reported patient satisfaction in the cTDR group versus the ACDF group. At 5 years, $96.4 \%$ of cTDR patients and $89.5 \%$ of ACDF patients reported being either very satisfied or somewhat satisfied with their treatment $(\mathrm{p}=0.04)$. At 5 years, $94.8 \%$ of patients in the cTDR group and $84.2 \%$ of patients in the ACDF group reported that they would definitely or probably recommend the surgery to a friend $(\mathrm{p}=0.01)$.

\section{Health-Related Quality of Life}

There was significant improvement versus baseline in SF-12 Physical Component Summary (PCS) and Mental Component Summary (MCS) scores in both groups. At 5 years (and at every time point), there was significantly more improvement in PCS scores in the cTDR patients (PCS Score 13) than ACDF patients (PCS Score 9; $\mathrm{p}=$ 0.0073) (Fig. 3 and Table 7). There were no statistically significant differences in MCS scores between groups.

\section{Subsequent Surgery}

The rate of investigator-reported nonunion was $14 \%$ (15 patients) in ACDF. The fusion rate assessed by radiographic success was $90.5 \%(95 / 105)$ at 5 years. Of the 15 patients reported by investigators as nonunion, 9 of these patients progressed to symptomatic nonunion that required subsequent surgical interventions due to fusion failure. This corresponds to a symptomatic nonunion rate of $8.6 \%$

Overall, there were fewer secondary surgeries in the cTDR group than in the ACDF group (cTDR 7.1\% [16/225] vs ACDF $21.0 \%$ [22/105]) $(\mathrm{p}=0.0006)$. There were signif- 
TABLE 2. Patient demographics and baseline characteristics: primary analysis population

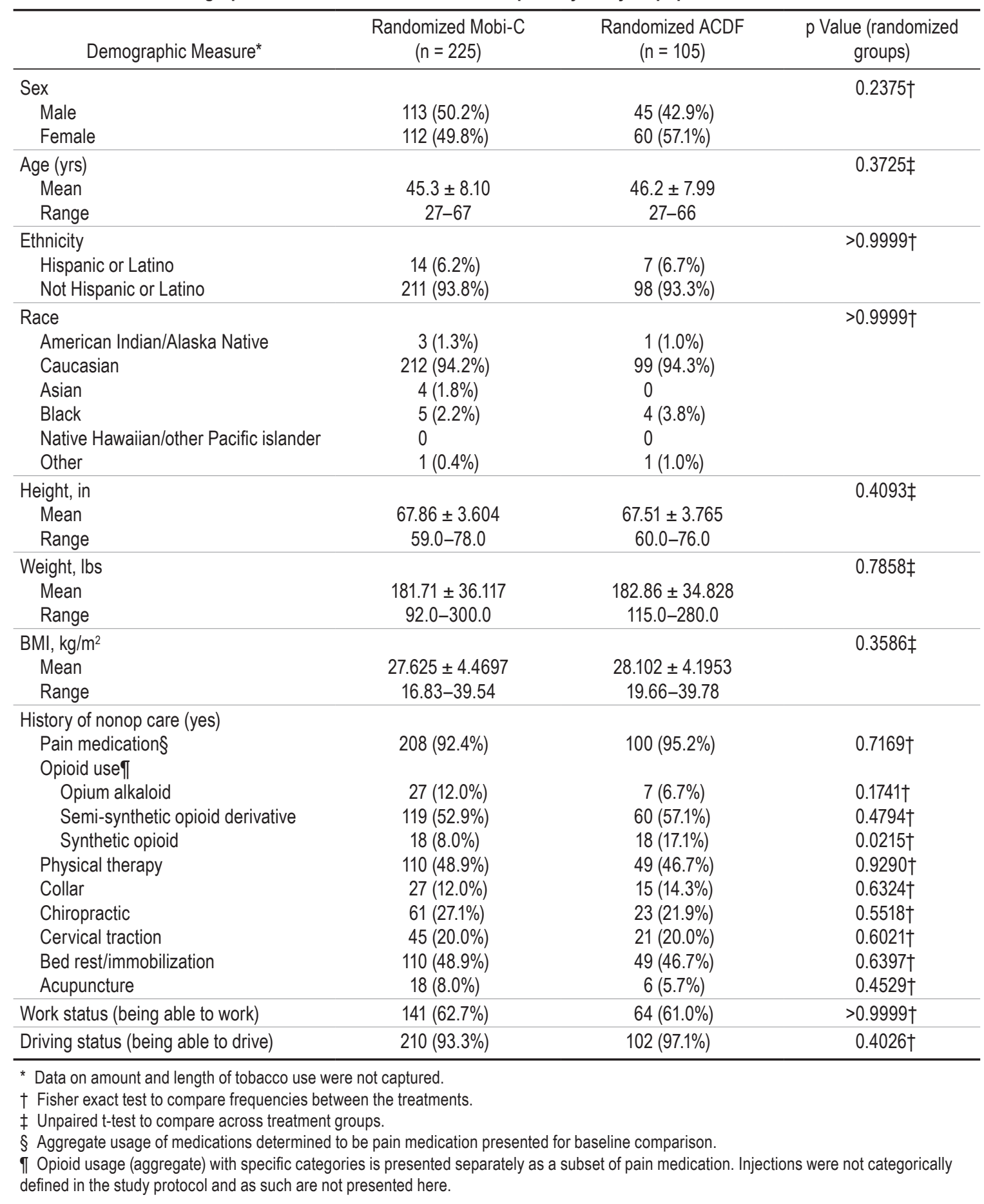

icantly fewer index-level secondary surgeries $(\mathrm{p}=0.0003)$ that were classified as study failures in the cTDR group (4\% [9/225]) than in the ACDF group (16.2\% [17/105]). Comparing mean reoperation-free survivorship (Fig. 4), cTDR patients demonstrate a significantly lower probability for secondary surgical intervention through 5 years ( $p$ $=0.0002$; log-rank test). The description of secondary surgery in both groups is provided in Appendix 2.

There were significantly more index-level reoperations in the ACDF group (16.2\%) than in the cTDR group
(4.3\%). There were also significantly more adjacent-level reoperations in the ACDF group (11.4\%) than in the cTDR group $(3.1 \%)$.

\section{Overall Study Success}

Using the FDA composite outcome measure for success, the overall success rates at 5 years were $61 \%$ and $31 \%$ for the cTDR and ACDF groups, respectively ( $\mathrm{p}<0.0001)$. The significantly higher overall success rate for the cTDR group meets superiority and noninferiority criteria. At 
TABLE 3. Preoperative evaluation of end points

\begin{tabular}{|c|c|c|c|}
\hline Variable & $\begin{array}{l}\text { Randomized Mobi-C } \\
\qquad(\mathrm{n}=225)\end{array}$ & $\begin{array}{l}\text { Randomized ACDF } \\
\quad(n=105)\end{array}$ & $\begin{array}{l}\text { p Value (randomized } \\
\text { groups) }\end{array}$ \\
\hline NDI & $53.86 \pm 15.576$ & $55.35 \pm 15.321$ & $0.4150^{*}$ \\
\hline VAS neck pain & $71.24 \pm 20.504$ & $74.56 \pm 18.937$ & $0.1619^{*}$ \\
\hline VAS left arm pain & $48.32 \pm 34.818$ & $49.92 \pm 33.799$ & $0.6948^{*}$ \\
\hline VAS right arm pain & $41.91 \pm 35.265$ & $45.64 \pm 35.440$ & $0.3726^{*}$ \\
\hline SF-12 PCS & $33.390 \pm 6.7184$ & $32.524 \pm 7.6635$ & $0.3051^{*}$ \\
\hline SF-12 MCS & $41.944 \pm 11.3041$ & $42.019 \pm 11.9173$ & $0.9564^{*}$ \\
\hline \multicolumn{4}{|c|}{ Normal neurological status $†$} \\
\hline Motor & $99(44.0 \%)$ & $54(51.4 \%)$ & $0.2363 \ddagger$ \\
\hline \multicolumn{4}{|l|}{ Sensory } \\
\hline Light touch & $110(48.9 \%)$ & $56(53.3 \%)$ & $0.4796 \ddagger$ \\
\hline Pinprick & $108(48.0 \%)$ & $52(49.5 \%)$ & $0.8140 \ddagger$ \\
\hline Reflexes & $80(35.6 \%)$ & $41(39.0 \%)$ & $0.5424 \ddagger$ \\
\hline Gait assessment§ & $215(95.6 \%)$ & $98(93.3 \%)$ & $0.5908 \ddagger$ \\
\hline \multicolumn{4}{|l|}{ Baseline ROM } \\
\hline \multicolumn{4}{|c|}{ Flexion-extension $\left({ }^{\circ}\right)$} \\
\hline Superior level & $9.13 \pm 4.849$ & $9.33 \pm 4.875$ & $0.7355^{*}$ \\
\hline Inferior level & $7.44 \pm 4.341$ & $7.14 \pm 3.860$ & $0.5574^{*}$ \\
\hline \multicolumn{4}{|l|}{ Baseline ROM } \\
\hline \multicolumn{4}{|l|}{ Lateral bending $\left(^{\circ}\right)$} \\
\hline Superior level & $5.76 \pm 3.374$ & $5.48 \pm 3.041$ & $0.4777^{*}$ \\
\hline Inferior level & $4.91 \pm 3.265$ & $4.77 \pm 2.866$ & $0.7227^{*}$ \\
\hline
\end{tabular}

each time point, there was a higher incidence rate of success in the cTDR group (Table 8). However, the differential in success rate between the 2 treatments increased following the 24-month follow-up interval. This is due to the decrease in the proportion of successful ACDF cases from 24 months of follow-up (42\%) to 60 months of follow-up (31\%) (Fig. 5).

\section{Potentially Related Adverse Events}

There was an increased percentage of all adverse events, treatment of emergent adverse events (i.e., those that occurred during the trial period, investigator-deemed adverse events, Clinical Event Committee-adjudicated adverse events, and related serious adverse events in the ACDF group at 5 years (Table 9). At the 5-year time point, the Clinical Event Committee determined that 10 patients in the cTDR group (4.4\%) and 9 patients in the ACDF group $(8.6 \%)$ had potentially device-related serious adverse events. There was no significant difference in the incidence of overall dysphagia and dysphonia complications in the

TABLE 4. Mean NDI score by time point

\begin{tabular}{|c|c|c|c|c|c|}
\hline \multirow[b]{2}{*}{ Time Point* } & \multicolumn{2}{|c|}{ Mean } & \multicolumn{2}{|c|}{ Standard Deviation } & \multirow[b]{2}{*}{ p Value $†$} \\
\hline & TDR & ACDF & TDR & ACDF & \\
\hline Baseline & 53.9 & 55.4 & 15.58 & 15.32 & \\
\hline Wk 6 & 25.7 & 34.3 & 17.69 & 18.05 & 0.0029 \\
\hline Mo 3 & 19.3 & 28.8 & 17.54 & 21.64 & 0.0009 \\
\hline Mo 6 & 17.9 & 25.1 & 17.38 & 19.53 & 0.0082 \\
\hline Mo 12 & 17.6 & 25.5 & 18.52 & 21.37 & 0.0034 \\
\hline Mo 18 & 18.4 & 25.7 & 18.24 & 21.2 & 0.0281 \\
\hline Mo 24 & 16.5 & 24 & 16.91 & 19.34 & 0.0032 \\
\hline Mo 36 & 16.5 & 26.9 & 17.82 & 21.41 & 0.0002 \\
\hline Mo 48 & 17.2 & 25.5 & 18.76 & 21.25 & 0.0048 \\
\hline Mo 60 & 16.8 & 26.4 & 17.43 & 20.37 & 0.0003 \\
\hline
\end{tabular}

* Both groups improved significantly at each time point compared to baseline.

$\dagger$ Unpaired t-test to compare change from baseline between groups, $p<0.05$. 


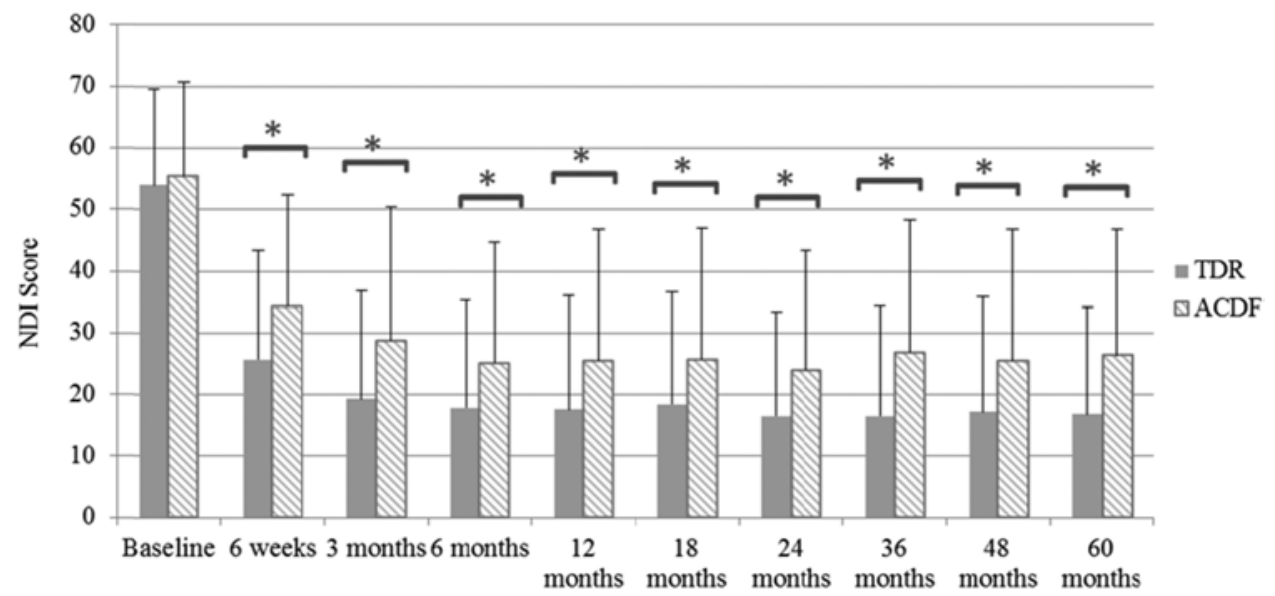

FIG. 2. Mean NDI score by time point. Error bars represent standard deviations. Both groups improved significantly at each time point compared with baseline. *Unpaired t-test to compare change from baseline between groups, $p<0.05$.

cTDR group (16\% of patients, $95 \%$ CI $11.4 \%-21.1 \%$ ) than in the ACDF group (21\% of patients, $95 \%$ CI $13.6 \%-30.0 \%$ ). There were 4 cases of implant malposition (suboptimal or undesired location) in the cTDR group (1.7\%).

\section{Radiological Outcomes}

cTDR patients maintained their baseline range of motion (ROM) in flexion/extension and lateral bending at both segments (Fig. 6). For cTDR patients, the mean ROM at the superior level was $10.1^{\circ} \pm 6.1^{\circ}$ in flexion/extension (baseline $9 \cdot 1^{\circ} \pm 4.8^{\circ}$ ). At the inferior level, the mean ROM was $8.4^{\circ} \pm 5.0^{\circ}$ in flexion/extension (baseline $7.4^{\circ} \pm 4.3^{\circ}$ ). At 60 months, lateral bending in cTDR patients was $5.6^{\circ} \pm$ $3.6^{\circ}$ in the superior level (baseline $5.8^{\circ} \pm 3.4^{\circ}$ ) and $5.1^{\circ} \pm$ $3.4^{\circ}$ in the inferior level (baseline $4.9^{\circ} \pm 3.3^{\circ}$ ).

Clinically relevant heterotopic ossification (Grade III or IV) was observed in $29.7 \%$ of cTDR patients with available radiographs at the 60 -month time point. cTDR patients presented with clinically relevant heterotopic ossification in $20.0 \%$ of superior levels and $21.6 \%$ of inferior levels. Grade IV heterotopic ossification was evident in at least 1 level in 9.7\% of cases.

Adjacent-segment degeneration was defined as an in- crease of 1 or more points of the Kellgren-Lawrence grading scale at 60 months in either segment when compared with baseline values. In patients who experienced degeneration at either level, cTDR patients had significantly less adjacent-segment degeneration than the ACDF group (50.7\% for cTDR vs $90.5 \%$ for ACDF, p < 0.0001). The superior levels at 60 months indicated significantly less degeneration in cTDR patients (32.6\%) than in ACDF patients $(70.8 \% ; \mathrm{p}<0.0001)$. Results for the inferior levels were similar (22.4\% for the cTDR group vs $55.1 \%$ for the ACDF group; $\mathrm{p}<0.0001)$.

\section{Neurological Status}

At 5 years, patients failing the neurological component of the FDA success criterion were $8.0 \%$ of cTDR patients $(n=18)$ and $5.7 \%$ of ACDF patients $(n=6)$; this difference was not significant. At 5 years, there were 18 instances of worsened muscle weakness (vs baseline) in the cTDR group and 12 instances of muscle weakness in the ACDF group.

\section{Discussion}

This is the first report of long-term outcome of 2-level cTDR from a US FDA IDE study. Two-level anterior cer-

TABLE 5. Mean VAS neck pain score by time point

\begin{tabular}{|c|c|c|c|c|c|}
\hline \multirow[b]{2}{*}{ Time Point* } & \multicolumn{2}{|c|}{ Mean } & \multicolumn{2}{|c|}{ Standard Deviation } & \multirow[b]{2}{*}{ p Value $†$} \\
\hline & cTDR & ACDF & CTDR & ACDF & \\
\hline Baseline & 71.24 & 74.56 & 20.50 & 18.94 & \\
\hline Wk 6 & 24.67 & 33.83 & 22.69 & 25.72 & 0.0822 \\
\hline Mo 3 & 19.57 & 31.89 & 23.99 & 30.29 & 0.0101 \\
\hline Mo 6 & 18.23 & 28.74 & 22.52 & 29.38 & 0.0303 \\
\hline Mo 12 & 19.77 & 29.11 & 25.92 & 29.91 & 0.0682 \\
\hline Mo 18 & 19.51 & 25.51 & 25.58 & 26.28 & 0.5366 \\
\hline Mo 24 & 16.59 & 20.45 & 24.15 & 23.95 & 0.6869 \\
\hline Mo 36 & 16.57 & 26.78 & 23.09 & 28.85 & 0.0517 \\
\hline Mo 48 & 17.74 & 25.44 & 24.62 & 28.32 & 0.2729 \\
\hline Mo 60 & 18.66 & 28.45 & 26.10 & 28.75 & 0.0743 \\
\hline
\end{tabular}

* Both groups improved significantly at each time point when compared to baseline.

$\dagger$ Unpaired t-test to compare change from baseline between groups, $p<0.05$. 
TABLE 6. Mean VAS arm pain score by time point

\begin{tabular}{cccccc}
\hline & \multicolumn{3}{c}{ Mean } & \multicolumn{2}{c}{ Standard Deviation } \\
\cline { 2 - 5 } Time Point & cTDR & ACDF & cTDR & ACDF & p Value \\
\hline Baseline & 68.74 & 72.74 & 25.01 & 21.64 & 0.5387 \\
\hline Wk 6 & 15.68 & 17.92 & 22.49 & 22.90 & 0.8052 \\
\hline Mo 3 & 15.58 & 20.52 & 23.96 & 27.63 & 0.4163 \\
\hline Mo 6 & 14.63 & 20.69 & 22.41 & 27.88 & 0.1419 \\
\hline Mo 12 & 14.22 & 21.73 & 23.14 & 27.10 & 0.6282 \\
\hline Mo 18 & 14.69 & 20.62 & 24.34 & 26.42 & 0.8663 \\
\hline Mo 24 & 11.91 & 16.16 & 19.46 & 21.89 & 0.4016 \\
\hline Mo 36 & 12.59 & 19.35 & 23.00 & 26.17 & 0.4868 \\
\hline Mo 48 & 13.38 & 20.63 & 22.72 & 27.44 & 0.1487 \\
\hline Mo 60 & 11.86 & 22.20 & 21.20 & 27.44 & \\
\hline
\end{tabular}

* The worst arm at baseline is followed. Both groups improved significantly at each time point when compared to baseline.

$\dagger$ Unpaired t-test to compare change from baseline between groups, $p<0.05$.

vical spine surgery, with cTDR or ACDF, is safe and effective in significantly improving patient outcome. Comparing CTDR and ACDF, disc replacement resulted in statistically significant greater improvement in general and disease-specific outcome measures compared with ACDF. Additionally, there was a lower incidence of index level and adjacent-level reoperation with cTDR.

Our results add to the growing body of literature that in the long term, cTDR results in improved clinical outcome versus ACDF in properly indicated patients who meet the inclusion and exclusion criteria described above. . $^{13,28-37,41}$ There was a statistically significant greater improvement in the NDI, SF-12, and patient satisfaction in the cTDR grop than in the ACDF. Overall, a large percentage of patients reported that they were satisfied with their treatments $(96.4 \%$ in the cTDR group, ACDF $89.5 \%$ in the ACDF group) or would undergo surgery again (94.8\% in the cTDR group, $84.2 \%$ in the ACDF group). Other studies (using other implant systems) have also established more improvement in outcome and lower rates of reoperation with cTDR compared with ACDF for treatment of singlelevel pathology. ${ }^{10,13,32,41}$ Not unexpectedly, the rate of major neurological adverse events and gait dysfunction was low in both study populations, confirming the safety of both procedures. In contrast to previous studies, ${ }^{21}$ there was no significant increase in dysphagia associated with ACDF at any time point in this study using a validated dysphagia outcome measure.

The rate and etiology of reoperation following surgical fusion remains controversial. The present study lends credence to the belief that fusion predisposes patients to reoperation. In the cTDR population, there was a statistically significant lower rate of overall subsequent surgeries (7\% in the cTDR group vs $21 \%$ in the ACDF group; $\mathrm{p}$ $=0.0006)$. The increased rate of subsequent surgery between the 2-year (11\%) and 5-year (16\%) end points in the ACDF population corresponded to changes in mean NDI score. The majority of the reoperations in both groups (9/16 cTDR patients vs 17/22 ACDF patients) involved the index level. The most common secondary surgery in the cTDR group at the index level was device removal. We surmise that in many cases the index-level device was removed to access or fixate adjacent-level pathology, not necessarily to correct pathology at the index level. Radiographic factors including motion may affect the outcome of the TDR. A full analysis of the radiographic predictors of secondary surgery, including clinical correlation, motion analysis, baseline degeneration, plate proximity to

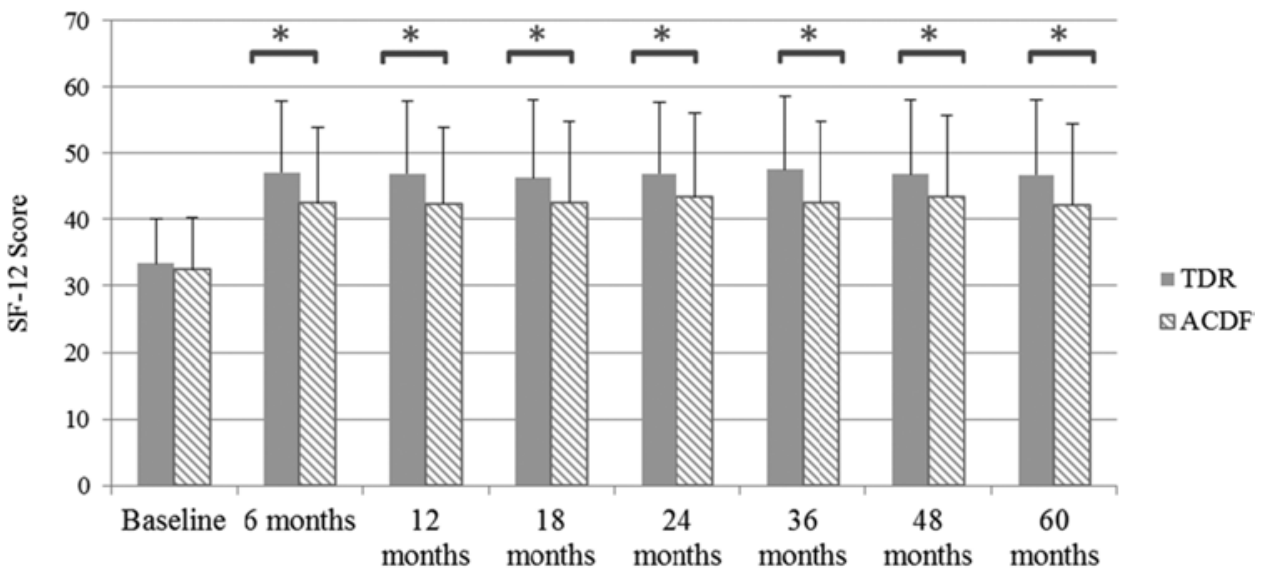

FIG. 3. Mean SF-12 PCS score by time point. Error bars represent standard deviations. Both groups improved significantly at each time point compared with baseline. *Unpaired t-test to compare change from baseline between groups, $p<0.05$. 
TABLE 7. Mean SF-12 PCS score by time point

\begin{tabular}{|c|c|c|c|c|c|}
\hline \multirow[b]{2}{*}{ Time Point* } & \multicolumn{2}{|c|}{ Mean } & \multicolumn{2}{|c|}{ Standard Deviation } & \multirow[b]{2}{*}{ p Value } \\
\hline & cTDR & ACDF & cTDR & ACDF & \\
\hline Baseline & 33.4 & 32.52 & 6.72 & 7.66 & \\
\hline Mo 6 & 47.1 & 42.52 & 10.78 & 11.35 & 0.0061 \\
\hline Mo 12 & 46.9 & 42.48 & 11.05 & 11.45 & 0.0090 \\
\hline Mo 18 & 46.3 & 42.54 & 11.77 & 12.20 & 0.0483 \\
\hline Mo 24 & 46.9 & 43.40 & 10.71 & 12.63 & 0.0333 \\
\hline Mo 36 & 47.6 & 42.65 & 10.97 & 12.19 & 0.0060 \\
\hline Mo 48 & 46.8 & 43.53 & 11.32 & 12.20 & 0.0459 \\
\hline Mo 60 & 46.8 & 42.20 & 11.32 & 12.26 & 0.0073 \\
\hline
\end{tabular}

* Both groups improved significantly at each time point compared to baseline.

$\dagger$ Unpaired t-test to compare change from baseline between groups, $p<0.05$.

endplates, and degree of heterotopic ossification, is outside the scope of this clinically oriented paper. ${ }^{31}$ The rate of investigator-reported nonunion in the ACDF population at any time point was $14 \%$ (15 patients). This rate of nonunion is comparable to the rates reported for 1-level ACDF with the ProDisc-C (13.1\% at 5 years) and the Prestige disc (10.0\% at 7 years)..$^{11,41}$ The rate of secondary surgery for symptomatic pseudarthrosis (8.6\%) is lower than historical estimates. ${ }^{39,40}$

Strengths of the study include the rigorous methodology involving prospective randomization with adequate statistical power. As a result of randomization, at baseline, the demographics and outcome scores were balanced between groups. Additionally, the 5-year follow-up rate was good in both groups, enabling adequate statistical power to test both noninferiority and superiority. Based on our analysis of follow-up, there is no evidence of any bias due to dropouts. The follow-up rates in our study for both Mobi$\mathrm{C}$ and $\mathrm{ACDF}$ are comparable or higher than those reported in other long-term randomized control trials of cervical arthroplasty. ${ }^{8,24,41}$ By utilizing an array of general and disease specific outcome measures and observing the effect (improved outcome in Mobi-C patients) in several different outcome measures (SF-12, NDI, satisfaction), we are more confident in our conclusion that 2-level cTDR was

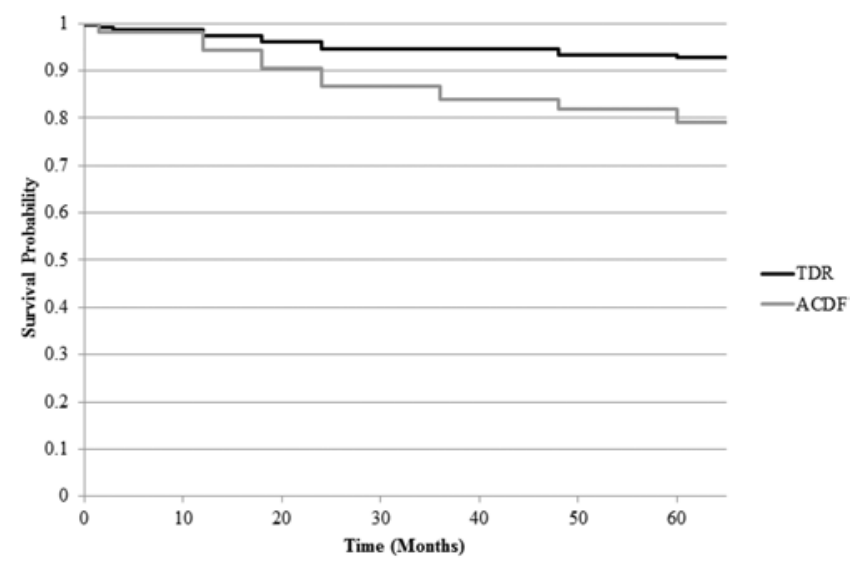

FIG. 4. Kaplan-Meier survival functions for subsequent surgical interventions in ACDF and TDR patients. TDR patients demonstrate a significantly different survival function than ACDF patients for subsequent surgical intervention through 60 months $(p=0.0002$, log-rank test). superior to ACDF than if the effect were only observed in a single instrument. Finally, in this case the data review was conducted using rigorous criteria in accord with FDA standards by independent investigators without any institutional or financial bias or prior involvement in the MobiC cTDR study.

Limitations of the study include the potential for affirmation bias on the part of the cTDR patients. Patients were not blinded to their treatment type since they may have had the opportunity to view their postoperative radiographs and they would have known about the presence or absence of a cervical collar for arthrodesis. It is possible that the improved outcome may be the result of affirmation bias. However, by 5 years we suspect that initial euphoria at inclusion into the treatment arm of a clinical trial may have subsided. Another limitation is the inherent subjectivity of the decision for reoperation. ${ }^{35}$ In this study, we believe that the reoperation rate represents an attempt to rescue deteriorating clinical outcomes, noting that the ACDF patient population experienced less NDI improvement, indicating a potential greater overall need for revision surgery. Each revision surgery was documented by the investigative site, including the related symptoms. A paper detailing the revision surgeries through 5 years is currently under review for publication. Early study definitions of success focused heavily on absence of neurological adverse events, since in the early days of cTDR there was some uncertainty among surgeons whether neurological adverse events would occur more frequently. Another limitation is that the racial distribution of the study population (94\% Caucasian) does not reflect that of the United States as a whole. We are

TABLE 8. Two-level overall success through 60 months

\begin{tabular}{cccc}
\hline Time Point & TDR & ACDF & Lower Bound \\
\hline Mo 6 & $140 / 219(63.9 \%)$ & $44 / 97(45.4 \%)$ & 0.0869 \\
\hline Mo 12 & $134 / 215(62.3 \%)$ & $43 / 95(45.3 \%)$ & 0.0706 \\
\hline Mo 18 & $126 / 213(59.2 \%)$ & $42 / 92(45.7 \%)$ & 0.0332 \\
\hline Mo 24 & $145 / 221(65.6 \%)$ & $42 / 99(42.4 \%)$ & 0.1347 \\
\hline Mo 36 & $137 / 202(62.9 \%)$ & $31 / 87(35.6 \%)$ & 0.1711 \\
\hline Mo 48 & $125 / 203(61.6 \%)$ & $29 / 89(32.6 \%)$ & 0.1908 \\
\hline Mo 60 & $124 / 204(60.8 \%)$ & $29 / 93(31.2 \%)$ & 0.1990 \\
\hline
\end{tabular}

* Lower bound $>0$ confirms superiority of TDR. 


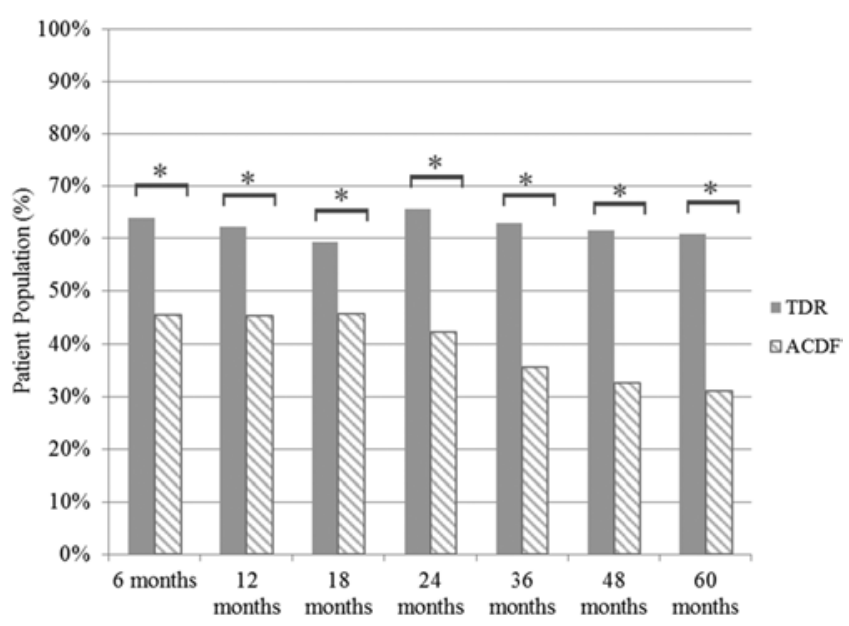

FIG. 5. Overall success through 60 months. Superiority of TDR over $\operatorname{ACDF}(p<0.025)$.

uncertain as to the reasons for this effect as the study was conducted at multiple centers. This finding may limit the generalizability of the results to non-Caucasian populations. All patients who were eligible for participation were offered the opportunity to participate in the trial. Other studies have also shown that minority participation in orthopedic trials is typically lower than would be expected based on census demographics. ${ }^{36}$ Without further information, it is also difficult to determine if a new sensory
TABLE 9. Percentage of population with Clinical Event Committee-adjudicated adverse events by time point through 60 months

\begin{tabular}{ccc}
\hline Time Point & TDR $(n=225)$ & ACDF $(n=105)$ \\
\hline Mo 6 & $1.3 \%$ & $1.0 \%$ \\
\hline Mo 12 & $2.7 \%$ & $2.9 \%$ \\
\hline Mo 18 & $3.1 \%$ & $6.7 \%$ \\
\hline Mo 24 & $3.6 \%$ & $6.7 \%$ \\
\hline Mo 36 & $4.0 \%$ & $6.7 \%$ \\
\hline Mo 48 & $4.0 \%$ & $7.6 \%$ \\
\hline Mo 60 & $4.4 \%$ & $8.6 \%$ \\
\hline
\end{tabular}

deficit that is remote from the index surgical event represents a de novo peripheral nerve entrapment syndrome or a new cervical spine pathology. We would assume that clinically significant physical examination changes causing new radiculopathy or myelopathy would be reflected in the outcome measures and reoperations. Additionally, the study protocol called for allograft fusion in the control group, similar to that of other IDE studies on disc replacement. ${ }^{23-28}$ We hypothesize that if an autograft were used, the reoperation rate might have been different; however, the use of an autograft has the potential for additional complications (such as donor site pain), and the decision not to use an autograft resulted in a well-matched control procedure to the cTDR group.
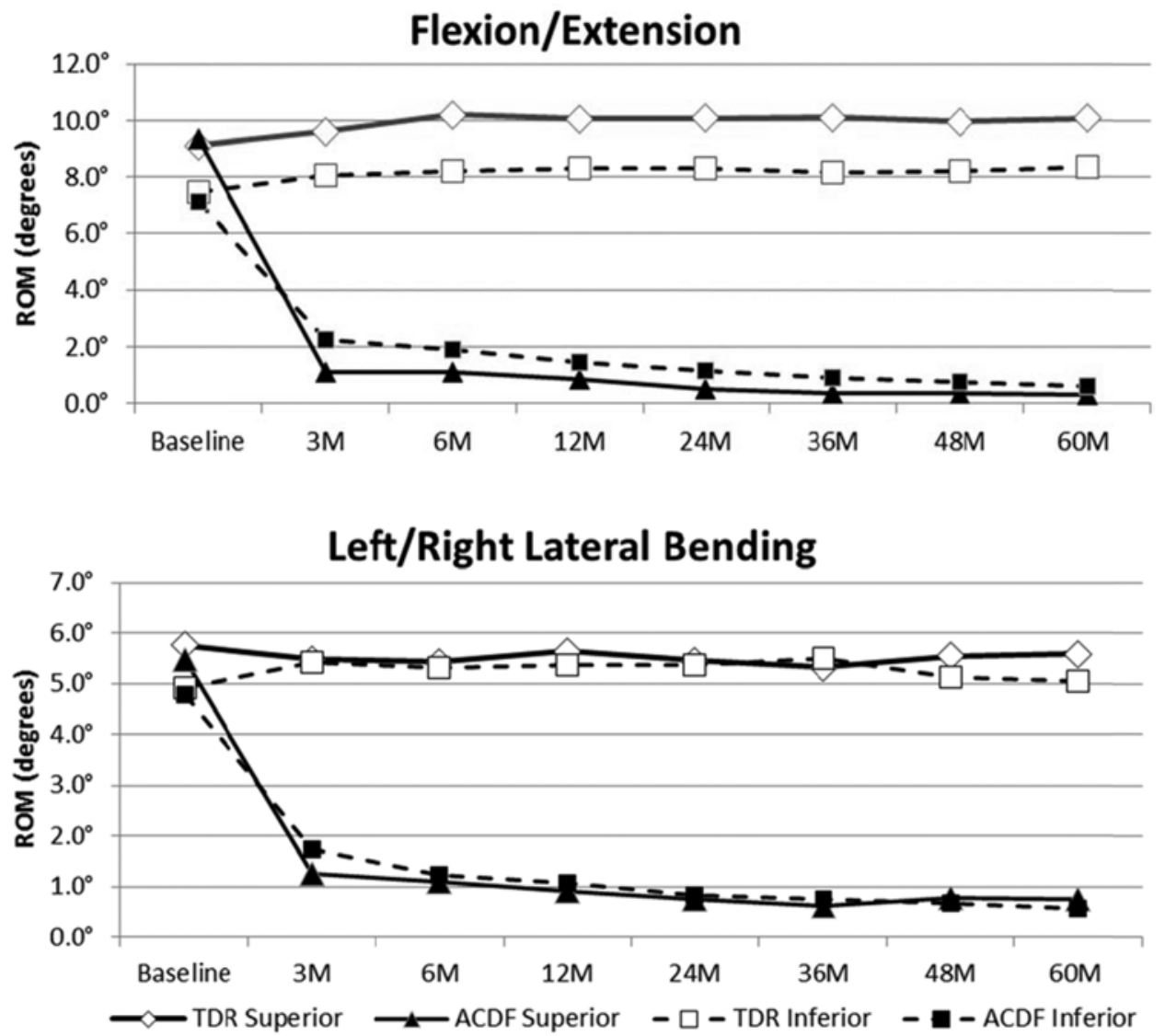

FIG. 6. ROM in both flexion/extension (upper) and left/right lateral bending (lower) through 60 months (M). 


\section{Conclusions}

We found a higher rate of success based on improvement in outcome and a lower rate of reoperation, at index and adjacent levels, among patients who were treated with a Mobi-C cTDR at 2 levels versus an ACDF at 5 years. It is important to consider that these results apply to the study population of patients with radiculopathy or myelopathy, objective muscle weakness, at least moderate to severe neck and arm pain, and concordant pathology at 2 contiguous levels in whom at least 6 weeks of conservative treatment has failed. In patients meeting the above criteria who are otherwise appropriate surgical candidates, 2-level cTDR was safe and effective with a lower rate of related adverse events compared with ACDF.

\section{Acknowledgments}

We would like to acknowledge the contribution of the study investigators at each of the 24 clinical sites across the US.

\section{References}

1. Anderson PA, Sasso RC, Hipp J, Norvell DC, Raich A, Hashimoto R: Kinematics of the cervical adjacent segments after disc arthroplasty compared with anterior discectomy and fusion: a systematic review and meta-analysis. Spine (Phila Pa 1976) 37 (22 Suppl):S85-S95, 2012

2. Auerbach JD, Anakwenze OA, Milby AH, Lonner BS, Balderston RA: Segmental contribution toward total cervical range of motion: a comparison of cervical disc arthroplasty and fusion. Spine (Phila Pa 1976) 36:E1593-E1599, 2011

3. Barrey C, Campana S, Persohn S, Perrin G, Skalli W: Cervical disc prosthesis versus arthrodesis using one-level, hybrid and two-level constructs: an in vitro investigation. Eur Spine J 21:432-442, 2012

4. Beaurain J, Bernard P, Dufour T, Fuentes JM, Hovorka I, Huppert J, et al: Intermediate clinical and radiological results of cervical TDR (Mobi-C) with up to 2 years of follow-up. Eur Spine J 18:841-850, 2009

5. Boselie TF, Willems PC, van Mameren H, de Bie R, Benzel EC, van Santbrink H: Arthroplasty versus fusion in singlelevel cervical degenerative disc disease. Cochrane Database Syst Rev 9:CD009173, 2012

6. Botelho RV, Moraes OJ, Fernandes GA, dos Santos Buscariolli Y, Bernardo WM: A systematic review of randomized trials on the effect of cervical disc arthroplasty on reducing adjacent-level degeneration. Neurosurg Focus 28(6):E5, 2010

7. Burkus JK, Haid RW, Traynelis VC, Mummaneni PV: Longterm clinical and radiographic outcomes of cervical disc replacement with the Prestige disc: results from a prospective randomized controlled clinical trial. J Neurosurg Spine 13:308-318, 2010

8. Burkus JK, Traynelis VC, Haid RW Jr, Mummaneni PV: Clinical and radiographic analysis of an artificial cervical disc: 7-year follow-up from the Prestige prospective randomized controlled clinical trial: Clinical article. J Neurosurg Spine 21:516-528, 2014

9. Cardoso MJ, Rosner MK: Multilevel cervical arthroplasty with artificial disc replacement. Neurosurg Focus 28:E19, 2010

10. Coric D, Nunley PD, Guyer RD, Musante D, Carmody CN, Gordon CR, et al: Prospective, randomized, multicenter study of cervical arthroplasty: 269 patients from the Kineflex|C artificial disc investigational device exemption study with a minimum 2-year follow-up: clinical article. J Neurosurg Spine 15:348-358, 2011
11. Cunningham BW, Hu N, Zorn CM, McAfee PC: Biomechanical comparison of single- and two-level cervical arthroplasty versus arthrodesis: effect on adjacent-level spinal kinematics. Spine J 10:341-349, 2010

12. Davis RJ, Kim KD, Hisey MS, Hoffman GA, Bae HW, Gaede $\mathrm{SE}$, et al: Cervical total disc replacement with the Mobi-C cervical artificial disc compared with anterior discectomy and fusion for treatment of 2-level symptomatic degenerative disc disease: a prospective, randomized, controlled multicenter clinical trial: clinical article. J Neurosurg Spine 19:532-545, 2013

13. Delamarter RB, Zigler J: Five-year reoperation rates, cervical total disc replacement versus fusion, results of a prospective randomized clinical trial. Spine (Phila Pa 1976) 38:711-717, 2013

14. Fallah A, Akl EA, Ebrahim S, Ibrahim GM, Mansouri A, Foote CJ, et al: Anterior cervical discectomy with arthroplasty versus arthrodesis for single-level cervical spondylosis: a systematic review and meta-analysis. PLoS One 7:e43407, 2012

15. Harrod CC, Hilibrand AS, Fischer DJ, Skelly AC: Adjacent segment pathology following cervical motion-sparing procedures or devices compared with fusion surgery: a systematic review. Spine (Phila Pa 1976) 37 (22 Suppl):S96-S112, 2012

16. Heller JG, Sasso RC, Papadopoulos SM, Anderson PA, Fessler RG, Hacker RJ, et al: Comparison of BRYAN cervical disc arthroplasty with anterior cervical decompression and fusion: clinical and radiographic results of a randomized, controlled, clinical trial. Spine (Phila Pa 1976) 34:101-107, 2009

17. Huppert J, Beaurain J, Steib JP, Bernard P, Dufour T, Hovorka I, et al: Comparison between single- and multi-level patients: clinical and radiological outcomes 2 years after cervical disc replacement. Eur Spine J 20:1417-1426, 2011

18. Jawahar A, Cavanaugh DA, Kerr EJ III, Birdsong EM, Nunley PD: Total disc arthroplasty does not affect the incidence of adjacent segment degeneration in cervical spine: results of 93 patients in three prospective randomized clinical trials. Spine J 10:1043-1048, 2010

19. Kepler CK, Brodt ED, Dettori JR, Albert TJ: Cervical artificial disc replacement versus fusion in the cervical spine: a systematic review comparing multilevel versus single-level surgery. Evid Based Spine Care J 3 (S1):19-30, 2012

20. Lawrence BD, Hilibrand AS, Brodt ED, Dettori JR, Brodke DS: Predicting the risk of adjacent segment pathology in the cervical spine: a systematic review. Spine (Phila Pa 1976) 37 (22 Suppl):S52-S64, 2012

21. McAfee PC, Cappuccino A, Cunningham BW, Devine JG, Phillips FM, Regan JJ, et al: Lower incidence of dysphagia with cervical arthroplasty compared with ACDF in a prospective randomized clinical trial. J Spinal Disord Tech 23:1-8, 2010

22. Murrey D, Janssen M, Delamarter R, Goldstein J, Zigler J, Tay B, et al: Results of the prospective, randomized, controlled multicenter Food and Drug Administration investigational device exemption study of the ProDisc-C total disc replacement versus anterior discectomy and fusion for the treatment of 1-level symptomatic cervical disc disease. Spine J 9:275-286, 2009

23. Park JH, Rhim SC, Roh SW: Mid-term follow-up of clinical and radiologic outcomes in cervical total disk replacement (Mobi-C): incidence of heterotopic ossification and risk factors. J Spinal Disord Tech 26:141-145, 2013

24. Phillips FM, Geisler FH, Gilder KM, Reah C, Howell KM, McAfee PC: Long-term outcomes of the US FDA IDE prospective, randomized controlled clinical trial comparing PCM cervical disc arthroplasty with anterior cervical discectomy and fusion. Spine (Phila Pa 1976) 40:674-683, 2015 
25. Phillips FM, Lee JY, Geisler FH, Cappuccino A, Chaput CD, DeVine JG, et al: A prospective, randomized, controlled clinical investigation comparing PCM cervical disc arthroplasty with anterior cervical discectomy and fusion. 2-year results from the US FDA IDE clinical trial. Spine (Phila Pa 1976) 38:E907-E918, 2013

26. Phillips FM, Tzermiadianos MN, Voronov LI, Havey RM, Carandang G, Dooris A, et al: Effect of two-level total disc replacement on cervical spine kinematics. Spine (Phila Pa 1976) 34:E794-E799, 2009

27. Pimenta L, McAfee PC, Cappuccino A, Cunningham BW, Diaz R, Coutinho E: Superiority of multilevel cervical arthroplasty outcomes versus single-level outcomes: 229 consecutive PCM prostheses. Spine (Phila Pa 1976) 32:13371344, 2007

28. Pracyk JB, Traynelis VC: Treatment of the painful motion segment: cervical arthroplasty. Spine (Phila Pa 1976) 30 (16 Suppl):S23-S32, 2005

29. Radcliff K, Zigler J, Zigler J: Costs of cervical disc replacement versus anterior cervical discectomy and fusion for treatment of single-level cervical disc disease: an analysis of the Blue Health Intelligence database for acute and long-term costs and complications. Spine (Phila Pa 1976) 40:521-529, 2015

30. Reitman CA, Hipp JA, Nguyen L, Esses SI: Changes in segmental intervertebral motion adjacent to cervical arthrodesis: a prospective study. Spine (Phila Pa 1976) 29:E221-E226, 2004

31. Rihn JA, Radcliff K, Hipp J, Vaccaro AR, Hilibrand AS, Anderson DG, et al: Radiographic variables that may predict clinical outcomes in cervical disk replacement surgery. J Spinal Disord Tech 28:106-113, 2015

32. Robertson JT, Papadopoulos SM, Traynelis VC: Assessment of adjacent-segment disease in patients treated with cervical fusion or arthroplasty: a prospective 2-year study. J Neurosurg Spine 3:417-423, 2005

33. Sasso RC, Anderson PA, Riew KD, Heller JG: Results of cervical arthroplasty compared with anterior discectomy and fusion: four-year clinical outcomes in a prospective, randomized controlled trial. J Bone Joint Surg Am 93:1684-1692, 2011

34. Sasso RC, Metcalf NH, Hipp JA, Wharton ND, Anderson PA: Sagittal alignment after Bryan cervical arthroplasty. Spine (Phila Pa 1976) 36:991-996, 2011

35. Singh K, Phillips FM, Park DK, Pelton MA, An HS, Goldberg EJ: Factors affecting reoperations after anterior cervical discectomy and fusion within and outside of a Federal Drug Administration investigational device exemption cervical disc replacement trial. Spine J 12:372-378, 2012

36. Somerson JS, Bhandari M, Vaughan CT, Smith CS, Zelle BA: Lack of diversity in orthopaedic trials conducted in the United States. J Bone Joint Surg Am 96:e56, 2014

37. Vaccaro A, Beutler W, Peppelman W, Marzluff JM, Highsmith J, Mugglin A, et al: Clinical outcomes with selectively constrained SECURE-C cervical disc arthroplasty: two-year results from a prospective, randomized, controlled, multicenter investigational device exemption study. Spine (Phila Pa 1976) 38:2227-2239, 2013

38. Verma K, Gandhi SD, Maltenfort M, Albert TJ, Hilibrand AS, Vaccaro AR, et al: Rate of adjacent segment disease in cervical disc arthroplasty versus single-level fusion: metaanalysis of prospective studies. Spine (Phila Pa 1976) 38:2253-2257, 2013

39. Wang JC, McDonough PW, Endow KK, Delamarter RB: A comparison of fusion rates between single-level cervical corpectomy and two-level discectomy and fusion. J Spinal Disord 14:222-225, 2001

40. Wang JC, McDonough PW, Endow KK, Delamarter RB: Increased fusion rates with cervical plating for two-level an- terior cervical discectomy and fusion. Spine (Phila Pa 1976) 25:41-45, 2000

41. Zigler JE, Delamarter R, Murrey D, Spivak J, Janssen M: ProDisc-C and anterior cervical discectomy and fusion as surgical treatment for single-level cervical symptomatic degenerative disc disease: five-year results of a Food and Drug Administration study. Spine (Phila Pa 1976) 38:203-209, 2013

\section{Disclosures}

The authors report the following. Dr. Radcliff: unpaid consultant for 4 Web Medical; board or committee member for ACSR; paid consultant for Altus Spine; paid consultant, research support, and unpaid consultant for DePuy, a Johnson \& Johnson Company; intellectual property (IP) royalties, paid consultant, and research support from Globus Medical; unpaid consultant for LDR; paid consultant and research support from Medtronic; other financial or material support from NEXXT Spine; other financial or material support from NuVasive; paid consultant for Orthofix, Inc.; IP royalties and paid consultant for Orthopedic Sciences, Inc.; research support from Pacira pharmaceuticals; research support from Paradigm Spine; and other financial or material support from Stryker. Dr. Albert: board or committee member for AAOS; board or committee member for American Orthopaedic Association; stock or stock options from ASIP; IP royalties from Biomet; stock or stock options from Biometrix; stock or stock options from Breakaway Imaging; stock or stock options from Crosstree; IP royalties and paid consultant for DePuy, a Johnson \& Johnson Company; paid consultant and stock or stock options from FacetLink; stock or stock options from Gentis; stock or stock options from InViVo Therapeutics; stock or stock options from Invuity; publishing royalties, financial or material support from Jay Pee; editorial or governing board Journal of Bone and Joint Surgery (American); stock or stock options in Paradigm Spine; stock or stock options in Pioneer; stock or stock options in PMIG; publishing royalties, financial or material support from Saunders/ Mosby-Elsevier; board or committee member for Scoliosis Research Society; editorial or governing board for Spine; editorial or governing board for Spine Deformity Journal; stock or stock options in Spinicity; publishing royalties, financial or material support from Thieme; other financial or material support from United Healthcare; and stock or stock options in Vertech. Dr. Coric: royalties, stock ownership, and consultant for Spine Wave; stock ownership in DiscGenics and Spinal Motion; and consultant for Medtronic, Globus Medical, and United Healthcare; and speaking and/or teaching arrangements for Globus.

\section{Author Contributions}

Conception and design: all authors. Acquisition of data: Coric. Analysis and interpretation of data: all authors. Drafting the article: Radcliff, Albert. Critically revising the article: all authors. Reviewed submitted version of manuscript: Radcliff, Coric. Approved the final version of the manuscript on behalf of all authors: Radcliff. Study supervision: Coric.

\section{Supplemental Information \\ Online-Only Content}

Supplemental material is available with the online version of the article.

Appendices 1 and 2. http://thejns.org/doi/suppl/10.3171/ 2015.12.SPINE15824.

\section{Correspondence}

Kris Radcliff, Department of Orthopedic Surgery, Thomas Jefferson University, Rothman Institute, 2500 English Creek Ave., Egg Harbor, NJ 08234. email: radcliffk@gmail.com. 